\title{
Fine Particles: Walter C. McCrone's Life-long Contribution to their Isolation, Identification and Use
}

\author{
Richard E. Bisbing* \\ *McCrone Associates, Inc., 850 Pasquinelli Drive, Westmont, Illinois 60559
}

We remember that Dr. Walter C. McCrone, Jr. started a laboratory and, fortunately for his colleagues, clients, and students, persevered with his laboratory with an extremely valuable toolthe polarizing light microscope, a remarkably useful approach to fine particle identification, and a powerful personality. His legacy to microscopy and microanalysis is: (1) the particle approach, (2) an emphasis on morphological analysis, and (3) a reliance on ultramicroanalysis and phase identification of fine particles to answer difficult questions and solve important problems.

Nearly a half-century ago (1956), Dr. McCrone opened and cultivated a very special microscopy and microanalysis laboratory. McCrone Associates, like all laboratories, has a special culture and, most importantly to its subsistence, a different culture than most contract laboratories. As Dr. McCrone intended, his laboratory was more than an inventory of analytical instruments or a group of technicians working for the technical manager; it has always been a consulting laboratory dedicated to using his approach to problem solving. One may think that there is magic involved because of the lore and mystique that now surround The McCrone Group following Dr. McCrone's high-profile projects such as the Vinland Map and the Turin Shroud. In fact, it does not matter what the fine particle problem is, the simple approach to the analysis and identification is the same: through modern microscopy, particles are isolated, characterized, and identified; and, based on their precise identity, their cause and origin can be inferred and documented.

Much of Dr. McCrone's original research following his Cornell University days, and during his time with Armour Research Foundation (1944-1959), was published as "crystallographic data" utilizing his beloved optical crystallography and fusion methods. His apparent metamorphosis from bureaucratic analytical chemist to consulting chemical microscopist is reflected in his articles in The Frontier, between 1945 and 1955. After his first retirement when he started McCrone Associates in 1956, his articles, chapters and books turned more toward fine particle identification and the singleminded task of promoting the use of polarized light microscopy (PLM) for a variety of applications, normally using particle identification as the key to success.

Dr. McCrone often had to explain how to identify single small particles: the microscopist uses morphological analysis, i.e., shape and optical characteristics either of the mote as a whole or of the significant component parts. He also explained that the particle approach requires particle manipulation. Dr. McCrone knew microscopists needed a picture book to help them identify particles they had not seen before, which led to The Particle Atlas, and he knew they needed supplies and tools for some of the manipulations, which led to McCrone Microscopes and Accessories, the instrument sales division of McCrone Associates, and McCrone Scientific, Ltd., London. In order to proselytize chemists to chemical microscopists he offered training in the industrial use of the polarizing microscope. In other words, he made a business out of recognizing thousands of particles and answering important questions with their identification--and he helped others do likewise. 
When morphological analysis using PLM is unsuccessful in identifying the particle of interest or unsuccessful in adequately characterizing its features to solve vital problems, the full complement of modern microscopical study and microanalysis can be brought to bear on the particle. In $1960 \mathrm{Dr}$. McCrone threw down the gauntlet defining the difference between trace analysis and ultramicroanalysis. He preached that microscopes are hard to beat when the sample is made up of a mixture of very tiny particles. If a sample contains a trace component dispersed as fine particles in a matrix or on a substrate, this becomes, with a microscope, a problem of ultramicroanalysis. Forty years later, he was still making his case in American Laboratory and The Microscope.

The understanding that fine particles can directly affect a product's safety or performance, or affect human health spurred the development of more sensitive ultramicroanalytical techniques. As microscopy and microanalysis expanded to meet this challenge, Dr. McCrone invited complementary and parallel specialists to join McCrone Associates. He knew that the laboratory would not profit on the basis of PLM alone. He knew that the only practical, long-term goal was atom-by-atom analysis. After PLM, the ultimate identification of fine particles came from his laboratory, where the research and consulting combined PLM with an ever-increasing sophistication in microanalysis. As Dr. McCrone preached PLM, his laboratory was enhancing instrumental microanalysis and routinely identifying particles using XRD, FTIR, Raman, SEM, EMA, TEM, SIMS, TIMS, and micro-beam instruments with lesser-known acronyms of past eras.

Over the last 45 years of Dr. McCrone's life, McCrone Associates, McCrone Research Institute, McCrone Environmental Services, McCrone Scientific, and former colleagues' or students' laboratories used his ideas and applied microscopy and microanalysis to fine particles from the moon to the earth, including: interplanetary dust, airborne particles from atomic weapon tests, volcanic dust in helicopter engines, make-up on deployed airbags, corrosion particles in cans, chemicals and parenterals, glass fibers from offices and homes, pigments from icons and artifacts, lead particles in soil, and dust from glacial cores. After more than 40,000 projects, it seems all the easy problems have already been solved, and those remaining are often contentious, litigious, and agonistic. Nevertheless, many fine particle problems will be solved in the $21^{\text {st }}$ century using analytical light microscopy, morphological analysis, ultramicroanalysis, and the particle approach, first used by Francois Vincent Raspail in the first half of the $19^{\text {th }}$ century and advocated throughout the last half of the 20th century by Dr. Walter C. McCrone. 